\title{
Effekte der Konzeption „Lesen durch Schreiben“ auf verschiedene Teilbereiche lauttreuer und orthografischer Verschriftung in der Mitte des ersten Schuljahres
}

\author{
Miriam Hess (D) Ann-Katrin Denn (D) Eva-Maria Kirschhock (D) \\ Alena Lorenz-Krause (ID Frank Lipowsky (iD
}

Online publiziert: 7. August 2020

(C) Der/die Autor(en) 2020

Zusammenfassung „Lesen durch Schreiben“ (Jürgen Reichen) ist eine Konzeption für den Schriftspracherwerb, in der die Kinder mit einer Anlauttabelle schreiben lernen, ohne dass eine Korrektur von Fehlern erfolgt. Anhand von differenzierten, qualitativen Fehleranalysen frei geschriebener Texte von 507 Kindern aus 31 ersten Klassen wird mehrebenenanalytisch untersucht, ob die Bedeutung, die diese Konzeption im Unterricht des ersten Schuljahres spielt, unter Kontrolle schriftsprachlicher Vorläuferfertigkeiten Effekte auf verschiedene Teilbereiche lauttreuer und orthografischer Verschriftung hat. Die Ergebnisse zeigen, dass die Kinder, in deren Unterricht „Lesen durch Schreiben“ laut Lehrperson eine größere Rolle spielt, insgesamt mehr Wörter falsch schreiben als Kinder, in deren Unterricht „Lesen durch Schreiben“ eine geringere Rolle spielt. Dieser Unterschied ist auf eine höhere Anzahl orthografisch falsch geschriebener Wörter zurückzuführen, während die Anzahl nicht lautge-

Dr. M. Hess $(\bowtie) \cdot$ Dr. E.-M. Kirschhock

Institut für Grundschulforschung, Universität Erlangen-Nürnberg, Regensburger

Str. 160, 90478 Nürnberg, Deutschland

E-Mail: miriam.hess@fau.de

Dr. E.-M. Kirschhock

E-Mail: eva-maria.kirschhock@fau.de

Dr. des. A.-K. Denn · Prof. Dr. F. Lipowsky

Fachgebiet für Empirische Schul- und Unterrichtsforschung, Universität Kassel,

Nora-Platiel-Str. 1, 34127 Kassel, Deutschland

Dr. des. A.-K. Denn

E-Mail: denn@uni-kassel.de

Prof. Dr. F. Lipowsky

E-Mail: lipowsky@uni-kassel.de

\section{A. Lorenz-Krause}

Studienseminar für Gymnasiallehramt Kassel, Franz-Treller-Str. 7, 34121 Kassel, Deutschland

E-Mail: fr.alena.lorenz@gmail.com 
treu verschrifteter Wörter in keinem systematischen Zusammenhang zur Rolle von „Lesen durch Schreiben“ in den jeweiligen Klassen steht.

Schlüsselwörter Lesen durch Schreiben - Schriftspracherwerb • Anfangsunterricht $\cdot$ Rechtschreibkompetenz $\cdot$ Unterrichtseffekte

\title{
Effects of the conception "Reading through Writing" on different subareas of phonetic and orthographic spelling in the middle of first grade
}

\begin{abstract}
Reading through writing" by Jürgen Reichen is a concept for written language acquisition in which children learn to write with an initial sound table. The conception is based on the fact that teachers do not correct written language errors. Freely written texts by 507 children from 31 first grade classrooms were evaluated on the basis of a differentiated, qualitative error analysis. Controlling for literacy skills at the beginning of school, multi-level analyses are carried out to determine whether the method has effects on different subareas of phonetic and orthographic spelling. The results show that children whose teachers report a higher importance of the conception "Reading through Writing" in their classroom make more spelling mistakes in total, which can be attributed to differences in orthographic spelling, whereas the number of words that are not correct in phonetic spelling does not differ significantly between the groups.
\end{abstract}

Keywords Reading through writing - Written language acquisition · Elementary teaching $\cdot$ Spelling skills $\cdot$ Effects of instruction

\section{Einleitung und Problemaufriss}

Lesen und Schreiben zu lernen hat für die gesamte Schulzeit und auch für das spätere Leben eine große Bedeutung. Deshalb verwundert es nicht, dass um die „richtige“ Methode der Vermittlung schon seit langem gerungen wird (Überblick bei Heuß 1993). Seit den 80er Jahren werden auch Unterrichtskonzeptionen im Schriftspracherwerb diskutiert, die sich an Maximen des „Offenen Unterrichts“ orientieren und insbesondere dem selbstgesteuerten Lernen des Kindes einen hohen Stellenwert zuschreiben. Eine Unterrichtskonzeption, die dabei häufig im Fokus der Auseinandersetzung steht, ist der Ansatz „Lesen durch Schreiben“ von Jürgen Reichen.

\section{Theoretischer und empirischer Hintergrund}

Reichens Ansatz „Lesen durch Schreiben“ gehört zu den deutschen Varianten des Spracherfahrungsansatzes (Kirschhock 2004). Seine Konzeption setzt als zentrales didaktisches Hilfsmittel von Anfang an die Anlauttabelle ein, um das selbstständige Schreiben eigener Wörter zu ermöglichen (Reichen 1988a, 1988b). Eine systematische Einführung in die Rechtschreibung hält Reichen im Hinblick auf die Entwick- 
lung einer Rechtschreibkompetenz nicht nur in der Anfangszeit, sondern insgesamt für kontraproduktiv (Reichen 2003, S. 131): ,Wer will, dass Kinder Rechtschreibkompetenz erwerben, muss [...] die Entwicklung der Rechtschreibung in Ruhe lassen, darf didaktisch nicht eingreifen (jedenfalls nicht direkt)“. Im Mittelpunkt seines Ansatzes steht die lautorientierte Verschriftung der Wörter. Kinder analysieren das Gesprochene und konstruieren daraus ihre Verschriftungen.

\subsection{Kennzeichen unserer Schrift und Strategien beim Schriftspracherwerb}

Da unsere Schrift keine reine Lautschrift ist, müssen Schreibanfängerinnen und Schreibanfänger lernen, bedeutungsunterscheidende Phoneme zu identifizieren, die sich nicht auf die eigene Artikulation, sondern auf ein abstraktes Lautschema beziehen, das der Schriftsprache zugrunde liegt (Bredel et al. 2011). Einem Phonem steht sprachstrukturell jeweils ein Graphem gegenüber. Komplex ist der Lernprozess für das Kind unter anderem auch deshalb, weil ein Phonem durch mehrere Grapheme repräsentiert werden kann (/v/ in Vase und Wasser). Die These, dass Kinder die Rechtschreibung erlernen, indem sie Wortbilder reproduzieren, gilt inzwischen als widerlegt (Scheerer-Neumann 1986). Kinder schreiben in der Anfangsphase unbekannte Wörter, indem sie diese nach ihrer Lautung abhören und jeweils neu konstruieren. Die Umsetzung dieses phonographischen Prinzips der Sprache ist ein erster, inklusiver Bestandteil auf dem Weg zum Orthografie-Erwerb, auch wenn dies oftmals zunächst zu „Fehlern“ im Sinne der rechtschriftlichen Norm führt, da die Lernenden ihre Strategien und Erkenntnisse erst allmählich dieser Norm angleichen (Augst und Dehn 2013; Scheerer-Neumann 2017).

National wie international werden diese Aneignungsprozesse in schriftsprachlichen Entwicklungsmodellen gefasst, die in der Regel drei aufeinanderfolgende Hauptstrategien beschreiben (Frith 1986): Als erste Annäherung wird meist die logographische Strategie dargestellt, in der sich die Kinder zunächst nur an visuellen Oberflächenmerkmalen der Schriftsprache orientieren. Ausdruck findet diese Strategie im Auswendiglernen von Zeichenfolgen wie dem eigenen Namen. In einer zweiten, von Frith (1986) als alphabetisch bezeichneten Strategie erkennen die Lernenden den Zusammenhang zwischen gesprochener und geschriebener Sprache. Wie oben beschrieben, müssen sie innerhalb dieser Strategie den Schritt von der phonetischen (an der eigenen Lautung ausgerichteten) Schreibung zur phonemischen Schreibung vollziehen (Thomé und Thomé 2019). Thomé und Thomé (2019) sprechen von Basisgraphemen für die jeweils häufigsten und zugleich orthografiesystematisch wichtigsten Schriftzeichen für ein Phonem. Diese Basisgrapheme als häufigste Verschriftungsform des Phonems decken etwa 90\% aller Einzelgrapheme ab. Das Basisgraphem für den Konsonanten /r/ ist beispielsweise das Graphem $<r>$, Orthographeme sind statistisch nicht so häufige Verschriftungen wie beispielsweise die Schreibweisen mit $<$ rr $>$ und $<$ rh $>$. Scheerer-Neumann (2010) gibt die Verschriftung des Gehörten mit Basisgraphemen als ein Ziel bis Ende des ersten Schuljahres an, wobei individuell große zeitliche Verschiebungen möglich sind. Nach Frith (1986) folgt auf die alphabethische Strategie dann die orthografische Strategie, in der zunehmend schriftsprachliche Strukturen, wie etwa die morphematischen, verstanden werden. Letzteres bedeutet, dass z. B. das Prinzip der Ableitungen vom Wortstamm 
verstanden wird, also dass das Wort „Häuser“ mit <äu> geschrieben wird, weil es sich auf „Haus“ beziehen lässt, und nicht mit <eu>, obwohl dies das Basisgraphem zum Laut /oI/ wäre.

Damit zusammenhängend rücken die selteneren Orthographeme in den Blick des Lerners, wie etwa das Graphem <ph> für das Phonem /f/ (Thomé und Thomé 2016). Zeitlich beginnt diese Strategie bei den meisten Kindern ab dem zweiten Schuljahr (Scheerer-Neumann 2010). Erstmals konnte die hierarchische Struktur der Kompetenzlevel (von einer unvollständigen lautorientierten Schreibung über eine lautgetreue Schreibung zur orthografisch korrekten Schreibung) pfadanalytisch in einer Studie von Jaeuthe et al. (2019) bestätigt werden.

\subsection{Forschungsstand zu Effekten von ,Lesen durch Schreiben“ auf die Rechtschreibleistung}

Reichens Konzept der intuitiven Aneignung der Orthografie wird von Fachdidaktik und -wissenschaft vielfach kritisiert (z.B. Corvacho del Toro und HoffmannErz 2014; Dürscheid 2011; Krauß 2014; Röber-Siekmeyer und Spiekermann 2000; Schründer-Lenzen 2007). Inwieweit sich „Lesen durch Schreiben“ (LdS) tatsächlich auf die Rechtschreibleistung im Anfangsunterricht auswirkt, wurde bereits mehrfach untersucht. Im Folgenden werden nur Ergebnisse aus Studien für die erste Jahrgangsstufe angeführt, die sich mit der Reichenkonzeption auseinandersetzen. In der einzigen existierenden Metaanalyse von Funke (2014) zu schriftsprachlichen Leistungen von Primarstufenkindern, die mit LdS unterrichtet wurden, wird deutlich, dass die Ergebnisse zu den Wirkungen des Konzepts von Reichen auf die Rechtschreibleistungen am Ende der ersten Jahrgangsstufe schwer interpretierbar sind, da die Befunde inkonsistent sind und nur bei zwei der fünf untersuchten Stichproben (Friedrich 2010; Weinhold 2006) die kognitiven Eingangsvoraussetzungen kontrolliert wurden. Sieht man sich einige der einbezogenen Studien im Detail an, werden Stärken und Herausforderungen in der Gegenüberstellung mit anderen methodischen Konzepten erkennbar. Brügelmann et al. (1994a, 1994b) verglichen kurz nach der Wende a) Schweizer Klassen am Ende des ersten Schuljahres, die nach „Lesen durch Schreiben“ unterrichtet worden waren, mit b) Klassen aus der ehemaligen DDR, in denen eine lehrgangsorientierte Fibelkonzeption mit einem hohen Anteil an Rechtschreibstunden verbunden wurde, und c) westdeutschen Klassen, in denen sowohl lehrgangsorientierter Unterricht mit einer Fibel stattfand als auch lernwegorientierte, offenere Zugänge zum Schriftspracherwerb umgesetzt wurden. Die Schweizer Kinder, die mit LdS unterrichtet worden waren, schrieben in einem Grundwortschatzdiktat am Ende des ersten Schuljahres $78 \%$ der Wörter richtig. Die Schülerinnen und Schüler der Stichprobe West kamen auf $63 \%$ richtig geschriebene Wörter und die Schülerinnen und Schüler aus der Stichprobe Ost hatten $81 \%$ geschriebene Wörter richtig.

In der Begleitstudie zum Hamburger Projekt „Elementare Schriftkultur“ (Hüttis-Graff 1997), die in der Metaanalyse nicht berücksichtigt wurde, da nach Funke (2014, S. 36) der Modellversuch andere Zielsetzungen als den Methodenvergleich hatte, fielen die Ergebnisse zuungunsten des Reichen-Ansatzes aus. Von 20 untersuchten ersten Klassen waren sechs der sieben Klassen, die am Ende des ersten 
Schuljahres am schlechtesten in einem Rechtschreibtest abschnitten, nach der Konzeption „Lesen durch Schreiben“ unterrichtet worden. Am Ende des ersten Schuljahres orthografisch richtig zu schreiben, wird von Reichen allerdings auch nicht angezielt. Einschränkend muss auch hier gesagt werden, dass - wie auch in der Studie von Brügelmann - keine Lernvoraussetzungen kontrolliert wurden.

In den Untersuchungen von May (2001), Schründer-Lenzen und Merkens (2006) sowie Weinhold (2006) gab es zwischen Fibelklassen und LdS-Klassen keine bedeutsamen und eindeutigen Entwicklungsunterschiede im Rechtschreiben bis zum Ende des ersten Schuljahres. Auch Hanke (2005), deren Stichprobe - wenn sie in ,offenen Ansätzen“ lernte - ebenfalls LdS praktizierte, ermittelte für das erste Schuljahr keine bedeutsamen Entwicklungsunterschiede im Rechtschreiben. In der neuesten und kontrovers diskutierten Studie (vgl. z. B. Brügelmann 2020) von Kuhl und RöhrSendlmeier (2018) wiesen die „Fibelklassen“ gegenüber Klassen, die nach Reichens Ansatz „Lesen durch Schreiben“ oder der „Rechtschreibwerkstatt“ unterrichtet wurden, Vorteile auf. Hier wurden 300 Erstklässler, nach Erhebung der Vorkenntnisse, in einem Längsschnitt bis zum vierten Schuljahr untersucht. Zusätzlich wurde eine querschnittliche Studie durchgeführt. Hauptergebnis des Vergleichs für den für die vorliegende Studie relevanten Zeitraum am Ende des ersten Schuljahres war ein Vorsprung der Fibelklassen in den Rechtschreibleistungen (vgl. ausführlich auch Kuhl 2020). In der Studie wurden allerdings die Leistungen in vorgegebenen Diktaten untersucht, sodass weiterhin offenbleibt, ob sich die Schreibleistungen in freien Texten unterscheiden. Auch eine differenzierte Analyse von Fehlerarten fand nicht statt.

\section{Fragestellung und Hypothesen}

Wie der bisherige Forschungsstand zeigt, sind die Ergebnisse zum Zusammenhang von „Lesen durch Schreiben“ und der Rechtschreibleistung im ersten Schuljahr uneinheitlich. Zudem basiert ein Teil der Studien auf wenig aussagekräftigen Untersuchungsdesigns (z. B. kleine Stichproben, fehlende Kontrolle von Eingangsleistungen, keine Berücksichtigung der Mehrebenenstruktur). Außerdem werden selten freie Texte untersucht und es wird in der Auswertung nicht differenziert auf einzelne Bereiche der Orthografie eingegangen.

In der vorliegenden Studie werden daher freie Texte von $N=507$ Erstklässlerinnen und Erstklässlern differenziert nach verschiedenen Bereichen lauttreuer und orthografischer Verschriftung ausgewertet, um anschließend den Einfluss der Konzeption LdS unter Kontrolle der Textlänge und zweier ausgewählter schriftsprachlicher Vorläuferfertigkeiten zu untersuchen.

Die übergeordnete Fragestellung lautet: Hat die Konzeption „Lesen durch Schreiben“" Effekte auf verschiedene Teilbereiche lauttreuer und orthografischer Verschriftung in der Mitte des ersten Schuljahres?

Folgende Hypothesen liegen den Analysen zugrunde: Unter Kontrolle von Textlänge und Vorläuferfertigkeiten machen die Lernenden in einem Unterricht, in dem die Konzeption „Lesen durch Schreiben“ eine stärkere Rolle spielt, im Vergleich zu Lernenden in einem Unterricht, in dem der Konzeption eine geringere Rolle zukommt, ... 
- auf Wortebene insgesamt mehr Fehler. Die höhere Fehleranzahl lässt sich dabei auf Fehler in der orthografischen Verschriftung, nicht aber auf Fehler in der lauttreuen Verschriftung zurückführen. (H1)

- auf Graphemebene insgesamt mehr Fehler. Die höhere Fehleranzahl lässt sich dabei auf Fehler in der orthografischen Verschriftung, nicht aber auf Fehler in der lauttreuen Verschriftung zurückführen. (H2)

- mehr Fehler in ausgewählten Teilbereichen orthografischer Verschriftung auf Graphemebene (Konsonanten- und Vokalverdopplungen, Verwendung von <ie> für /i:/ und von Schwa-Lauten am Ende von flektierten Verben). (H3)

- mehr Fehler im Bereich der Groß-und Kleinschreibung. (H4)

- mehr Fehler im Bereich der Getrennt- und Zusammenschreibung. (H5)

- mehr Fehler im Bereich der Interpunktion. (H6)

\section{Methode}

\subsection{Angabe der Lehrpersonen zur Unterrichtskonzeption, ,Lesen durch Schreiben"6}

In der Längsschnittstudie PERLE (Persönlichkeits- und Lernentwicklung von Grundschulkindern, vgl. Lipowsky et al. 2013) wurden die Lehrpersonen der teilnehmenden Klassen mittels Fragebogen zu Beginn des ersten Schuljahres gebeten, die Rolle, die LdS in ihrem Unterricht spielt, auf einer vierstufigen Skala einzuschätzen: „In meinem Unterricht spielt die Methode, Lesen durch Schreiben “von J. Reichen (1) gar keine Rolle, (2) eine untergeordnete Rolle, (3) eine wichtige Rolle oder (4) eine dominante Rolle“. Von den 31 in die Analysen einbezogenen Lehrpersonen geben 18 (58,10\%) an, die Konzeption spiele in ihren Klassen keine oder nur eine untergeordnete Rolle. Diese Lehrpersonen berichten auch alle, eine Fibel zu nutzen. Insgesamt 13 Lehrkräfte $(42,00 \%)$ geben an, dass die Konzeption „Lesen durch Schreiben“ eine wichtige oder dominante Rolle in ihrem Unterricht spielt. Von diesen Klassen berichten dennoch zehn Lehrpersonen von der Verwendung einer Fibel, während drei Lehrpersonen angeben, keine Fibel zu nutzen (Tab. 1).

Neben der Angabe zur Fibelnutzung wurden den Deutschlehrpersonen der PERLE-Studie auch weitere Fragen zum schriftsprachlichen Unterricht gestellt, von denen ausgewählte Items in Tab. 2 mit der Angabe zur Rolle von LdS korreliert wurden, um die Angaben der Lehrpersonen zur Rolle von LdS zu validieren. Die Lehrpersonen sollten die Fragen auf einer Skala von 1 ,stimmt gar nicht“ bis 4 ,stimmt genau“ beantworten. Dabei zeigt sich, dass Lehrpersonen, welche die Bedeutung von LdS für ihren Unterricht höher einschätzen, seltener Rechtschreibfehler unmittelbar verbessern $(r=-0,69 ; p \leq 0,001)$ und häufiger Rechtschreibfehler zulassen, um den Lernstand der Kinder erkennen zu können $(r=0,39 ; p \leq 0,05)$. Die Variable zur Einschätzung der Rolle des Konzepts „Lesen durch Schreiben“ zeigt somit erwartungskonforme Zusammenhänge mit anderen Angaben der Lehrpersonen, was auf die Validität der Angaben zur Rolle von „Lesen durch Schreiben“ deutet (vgl. auch Hess et al. in Vorbereitung). 
Tab. 1 Angaben der Lehrpersonen zur Bedeutung der Rolle von „Lesen durch Schreiben“ $(N=31$ Lehrpersonen)

\begin{tabular}{lllll}
\hline $\begin{array}{l}\text { Einschätzung der Rolle „Lesen } \\
\text { durch Schreiben“ }\end{array}$ & $\begin{array}{l}\text { Anzahl der } \\
\text { Klassen }\end{array}$ & $\begin{array}{l}\text { Prozentualer } \\
\text { Anteil (\%) }\end{array}$ & Verwendung einer Fibel \\
\hline Keine Rolle & 12 & 38,70 & Ja & 12 \\
& & & Nein & 0 \\
Untergeordnete Rolle & 6 & 19,40 & Ja & 6 \\
& \multirow{2}{*}{11} & 35,50 & Nein & 0 \\
Wichtige Rolle & & & Ja & 10 \\
& 2 & 6,50 & Nein & 1 \\
Dominante Rolle & & & Ja & 0 \\
& & & Nein & 2 \\
\hline
\end{tabular}

Tab. 2 Deskriptive Statistiken zu Angaben der Lehrpersonen zum schriftsprachlichen Unterricht und Korrelationen zur Rolle von LdS

\begin{tabular}{lllllll}
\hline Item & Min & Max & $M$ & $S D$ & $r$ & $p$ \\
\hline $\begin{array}{l}\text { (1) Rechtschreibfehler lasse ich die Kinder } \\
\text { unmittelbar verbessern, damit sie sich }\end{array}$ & 1 & 4 & 2,45 & 0,77 & $-0,69$ & $\leq 0,001$ \\
nicht einprägen & & & & & & \\
$\begin{array}{l}\text { (2) Ich lasse Rechtschreibfehler zu, weil } \\
\text { sie mir zeigen, auf welchem Stand sich die }\end{array}$ & 1 & 4 & 2,94 & 0,85 & 0,39 & 0,029 \\
$\begin{array}{l}\text { Kinder beim Erwerb der Schriftsprache } \\
\text { befinden }\end{array}$ & & & & & & \\
\hline
\end{tabular}

\subsection{Die Schülertexte und deren Auswertung}

Für die Analysen dieses Beitrags werden Daten der Videostudie Deutsch verwendet, die im Rahmen des Projekts PERLE in der Mitte des ersten Schuljahres stattfand (Lotz und Corvacho del Toro 2013). Um den Unterricht inhaltlich vergleichbar zu halten, wurden alle Lehrpersonen aufgefordert, eine ca. 90-minütige Unterrichtsstunde zu gestalten, in der das Bilderbuch „Lucy rettet Mama Kroko“ (Doucet und Wilsdorf 2005) im Mittelpunkt steht. Darin geht es um das Mädchen Lucy, das bei einer Krokodilfamilie aufwächst, aber aufgrund ihrer Andersartigkeit von den anderen Krokodilen gehänselt wird. Am Ende des ersten Teils beschließt Lucy deshalb, Mama Kroko und die Krokodilfamilie zu verlassen und ihre richtige Familie zu suchen. Als verpflichtende Unterrichtsinhalte wurde u.a. das Schreiben eines Abschiedsbriefs an Mama Kroko vorgegeben.

Es wurden hier nur diejenigen Texte einbezogen, die als Briefe klassifiziert werden konnten. Nicht als Brief gewertet wurden bspw. Zeichnungen oder Aneinanderreihungen von Buchstaben ohne erkennbaren Zusammenhang (Kürzinger et al. 2013). Für die vorliegende Untersuchung wurde eine von insgesamt 33 Klassen ausgeschlossen, da der Brief ein Gemeinschaftsprodukt der Klasse war, und eine Klasse, da die Briefe in Partnerarbeit verfasst wurden. Die Stichprobe dieses Beitrags umfasst somit 507 Kinder (54\% weiblich und 46\% männlich) aus 31 Klassen.

Da den Lehrkräften die didaktisch-methodische Aufbereitung der Unterrichtsinhalte freigestellt wurde, hatten die Schülerinnen und Schüler unterschiedlich viel 
Zeit für die Phase des Briefschreibens. Die Länge der Texte unterscheidet sich auch deshalb deutlich zwischen den Kindern: Es gibt Briefe, die im Minimum aus drei und im Maximum aus 103 Wörtern $(M W=17,03 ; S D=9,82)$ bestehen. Aufgrund dieser Varianz - und da die Anzahl von Fehlern natürlich abhängig von der Wortanzahl ist - wird die Textlänge in den Analysen dieses Beitrags als Kontrollvariable einbezogen. Im Mittel werden 1,94 Sätze (Min=1; Max =10; $S D=1,25)$ geschrieben. Durchschnittlich sind 16,74 Wörter identifizierbar $(\operatorname{Min}=3 ; \operatorname{Max}=103 ; S D=9,79)$. Nicht lesbare Wörter wurden nicht in die Analysen einbezogen, da bei diesen keine Analyse der orthografischen Fehler erfolgen kann. Die 507 Schülerinnen und Schüler schrieben insgesamt 145 nicht lesbare Wörter $(M=0,29 ; \operatorname{Min}=0 ; \operatorname{Max}=10$; $S D=0,93)$. Mehrebenenanalysen unter Kontrolle der Textlänge und der Vorläuferfertigkeiten Anlaute hören und Buchstabenkenntnis zeigen keinen systematischen Zusammenhang zwischen der Anzahl nicht identifizierbarer Wörter und der Rolle der Reichen-Methode im Unterricht.

Die vorab transkribierten Schülertexte (vgl. Kürzinger et al. 2013) wurden anhand eines detaillierten Fehlerkatalogs (vgl. Tab. 3) auf Basis der Instrumente OLFA 1-2 (Thomé und Thomé 2011) und OLFA 3-9 (Thomé und Thomé 2010) auf Graphemebene sowie anhand des Salzburger Lese- und Rechtschreibtests (SLRT; Landerl et al. 2006) auf Wortebene ausgewertet (ausführlich vgl. Lorenz 2017).

Mit der Oldenburger Fehleranalyse (OLFA) kann die orthografische Kompetenz auf Basis orthografietheoretischer, entwicklungspsychologischer und psycholinguistischer Grundlagen anhand freier Schülertexte ermittelt werden. Grundlegend ist die Unterscheidung von Basis- und Orthographemen und die daran anknüpfende qualitative Fehleranalyse auf Ebene der einzelnen Grapheme, die orthografische, lautgetreue, annähernd lautgetreue und unsystematische (nicht lautgetreue) Schreibungen unterscheidet (Thomé und Thomé 2011).

Der SLRT ist ursprünglich ein Lückendiktat, bei dem die Fehler anschließend in $\mathrm{N}$-Fehler (Wort ist nicht lauttreu verschriftet) und O-Fehler (Wort ist zwar lautgetreu, aber nicht orthografisch korrekt geschrieben) kategorisiert werden. Im Gegensatz zur OLFA findet die Auswertung hier also auf Wortebene statt.

Ursprünglich wurden noch weitere Fehlerkategorien unterschieden und kodiert. Diese sind aber teilweise sehr spezifisch (z. B. ,e/eu für ä/äu und umgekehrt“, ,,w für v und umgekehrt"). Daher wurden nur diejenigen Kategorien in die Analysen aufgenommen, die sowohl inhaltlich bedeutsam erschienen als auch eine ausreichend hohe Varianz aufwiesen (für nähere Informationen zu weiteren Kategorien vgl. Lorenz 2017).

Die Auswertungen wurden von einer Sprachwissenschaftlerin durchgeführt, aber in regelmäßigen Abständen konsensuell validiert und stichprobenartig überprüft. Eine post-hoc-Überprüfung der Beurteilerübereinstimmungen, in deren Rahmen aus jeder Klasse ein per Zufallsziehung ausgewählter Text von zwei weiteren Kodiererinnen unabhängig voneinander ausgewertet wurde, ergab für die einzelnen Fehlerkategorien paarweise prozentuale Übereinstimmungen zwischen $P \ddot{U}=91,43 \%$ und $P \ddot{U}=100,00 \%$, wobei die prozentuale Übereinstimmung im Mittel bei $P \ddot{U}=99,29 \%$ liegt. 
Tab. 3 Fehlerkategorien und -beispiele für die vorliegende Studie (vgl. Lorenz 2017, S. 71-77)

\begin{tabular}{|c|c|c|c|}
\hline Kategorien & & Beispiel & Quelle \\
\hline 1 & Fehler auf Wortebene & & \\
\hline 1.1 & $\begin{array}{l}\text { Anzahl der richtig geschriebenen Wörter (ohne } \\
\text { Groß-/Kleinschreibung) }\end{array}$ & - & Eigenentwicklung \\
\hline 1.2 & $\begin{array}{l}\text { Anzahl der „O-Fehler“ (lauttreue, aber nicht } \\
\text { orthografisch korrekte Wörter) }\end{array}$ & Tak (Tag) & SLRT \\
\hline 1.3 & $\begin{array}{l}\text { Anzahl der „N-Fehler“ (nicht lauttreu ver- } \\
\text { schriftete Wörter) }\end{array}$ & Aag (Tag) & SLRT \\
\hline 2 & OLFA-Stufen & & \\
\hline 2.1 & Fehleranzahl OLFA 1-2 & - & OLFA $1-2$ \\
\hline 2.2 & Fehleranzahl OLFA 1-2 (Stufe I) & - & OLFA $1-2$ \\
\hline 2.3 & Fehleranzahl OLFA 1-2 (Stufe II) & - & OLFA $1-2$ \\
\hline 2.4 & Fehleranzahl OLFA 1-2 (Stufe III) & - & OLFA $1-2$ \\
\hline 3 & Ausgewählte orthografische Fehler & & \\
\hline 3.1 & $\begin{array}{l}\text { Einfachschreibung für Konsonantenverdoppe- } \\
\text { lung }\end{array}$ & imer (immer) & OLFA $1-2$ \\
\hline 3.2 & $\begin{array}{l}\text { Einfachschreibung für markierte Vokallänge } \\
\text { (außer i für ie) }\end{array}$ & $\operatorname{ser}(\operatorname{seh} r)$ & OLFA $1-2$ \\
\hline 3.3 & i für ie bei langem /i:/ & libe (liebe) & OLFA $1-2$ \\
\hline 3.4 & ie für i bei langem /i:/ & $\begin{array}{l}\text { Krokodiel } \\
\text { (Krokodil) }\end{array}$ & OLFA $1-2$ \\
\hline 3.5 & $\begin{array}{l}\text { Fehlender Schwa-Laut am Ende eines Verbs } \\
\text { 1. Person Singular Präsens }\end{array}$ & ich mach (mache) & Eigenentwicklung \\
\hline 4 & Groß- und Kleinschreibung & & \\
\hline 4.1 & Klein- für Großschreibung & mama (Mama) & OLFA $3-9$ \\
\hline 4.2 & Groß- für Kleinschreibung & Ich Bin (bin) & OLFA 3-9 \\
\hline 4.3 & Großschreibung im Wort & MaMa (Mama) & OLFA 3-9 \\
\hline 5 & Getrennt- und Zusammenschreibung & & \\
\hline 5.1 & Getrennt- für Zusammenschreibung & $\begin{array}{l}\text { Haus Boot } \\
\text { (Hausboot) }\end{array}$ & OLFA 3-9 \\
\hline 5.2 & Zusammen- für Getrenntschreibung & $\begin{array}{l}\text { dieganze } \\
\text { (die ganze) }\end{array}$ & OLFA 3-9 \\
\hline 5.3 & $\begin{array}{l}\text { Getrenntschreibung von unselbstständigen } \\
\text { Teilen }\end{array}$ & da rum (darum) & Eigenentwicklung \\
\hline 5.4 & Platzhalter zwischen Wörtern fehlt & ichwoltedirsagen & Eigenentwicklung \\
\hline 6 & Interpunktion & & \\
\hline 6.1 & Fehleranzahl Satzabschlusszeichen gesamt & $\begin{array}{l}\text { Ich gehe weg } \\
\text { (Punkt) }\end{array}$ & OLFA 3-9 \\
\hline 6.2 & Fehleranzahl Satzmittezeichen gesamt & $\begin{array}{l}\text { Ich gehe weil } \\
\text { (Komma) }\end{array}$ & OLFA 3-9 \\
\hline 6.3 & Fehleranzahl Interpunktion gesamt & - & OLFA 3-9 \\
\hline
\end{tabular}




\subsection{Vorläuferfertigkeiten Schriftspracherwerb}

Um bei der Untersuchung der Effekte der Konzeption LdS die individuellen Vorläuferfertigkeiten zu kontrollieren, werden sowohl die phonologische Bewusstheit als auch die Buchstabenkenntnis der Schülerinnen und Schüler in die Analysen miteinbezogen. Dazu werden die Subtests „Anlaute hören“ und „Buchstaben lesen“ des Lest 4-7 (Moser et al. 2004') herangezogen. Die Lernenden wurden darin mit acht Bildern, deren Anlaute sie nennen sollten (bspw. „F“ für „Flöte“) und 20 Buchstaben konfrontiert, die von ihnen benannt werden sollten. Bei beiden Skalen wurde eine Rasch-Skalierung durchgeführt und WLE-Schätzer als Personenfähigkeitsparameter ausgelesen (Gresser et al. 2009), die für die Analysen genutzt werden. Zur besseren Interpretierbarkeit werden hier die deskriptiven Rohwerte der Variablen dargestellt (,Anlaute hören“: $M=5,93 ; \operatorname{Min}=0 ; \operatorname{Max}=8 ; S D=2,09$ und „Buchstaben lesen“: $M=10,50 ;$ Min = 0; $M a x=19 ; S D=5,59)$. Beide Skalen erwiesen sich in Vorab-Analysen als starke Prädiktoren für die Rechtschreibleistung zum Ende des ersten Schuljahres (Lotz et al. 2013).

\subsection{Analysemethoden}

Die Vorläuferfertigkeiten (UV) sowie die Anzahl und die Art von Fehlern beim Verschriften des Lucy-Texts (AV) gehen als Individualmerkmale in die Analysen ein, während die Rolle des Konzepts „Lesen durch Schreiben“(UV) ein Klassenmerkmal darstellt. Bei der Analyse von Unterrichtsmerkmalen und deren Zusammenhängen mit Merkmalen auf Individualebene wird daher diese hierarchische Datenstruktur (Ditton 1998) durch die Spezifizierung von Mehrebenenmodellen mit dem Programm Mplus (Muthén und Muthén 1998-2012) berücksichtigt.

Fehlende Werte liegen in den Daten nur für die Vorläuferfähigkeiten der Schülerinnen und Schüler vor. Insgesamt fehlen die Angaben von 13 Kindern, die an den Testtagen verhindert waren. Für die Mehrebenenanalysen werden nur Fälle verwendet, in denen auf allen Variablen gültige Werte vorliegen $(N=494$ Schülerinnen und Schüler aus $N=31$ Klassen).

\section{Ergebnisse}

Im Folgenden werden zunächst immer die deskriptiven Statistiken dargestellt (Min= Minimalwert; Max = Maximalwert; $M=$ Mittelwert; $S D=$ Standardabweichung; $I C C=$ Intraclass-Korrelation). Darauf folgen die Ergebnisse der Mehrebenenanalysen. Die abhängigen Variablen finden sich jeweils in der ersten Zeile und werden entsprechend der vorherigen Tabellen mit den deskriptiven Statistiken nummeriert. Auf Ebene 1 (E1) werden immer die Textlänge (vgl. Abschn. 4.2) sowie die Vorläuferfertigkeiten „Anlaute hören“ und „Buchstabenkenntnis“ (vgl. Abschn. 4.3) kontrolliert. Als unabhängige Variable auf Ebene 2 (E2) wird die Einschätzung

\footnotetext{
${ }^{1}$ Moser, U., Berweger, S. \& Lüchinger-Hutter, L. (Hrsg.). (2004). Lest 4-7. Lern- und Entwicklungsstand bei 4- bis 7-Jährigen. Unveröffentlichter Test.
} 
der Lehrperson zur Rolle von LdS für ihren Unterricht verwendet. Höhere Werte bedeuten hier eine größere Rolle der Reichen-Konzeption (vgl. Abschn. 4.1). Für jedes Modell werden die Werte $\beta$ (standardisierter Regressionskoeffizient), die Irrtumswahrscheinlichkeit $p(* * * p \leq 0,001 ; * * p \leq 0,01 ; * p \leq 0,05)$ und die Varianzaufklärung $\mathrm{R}^{2}$ aufgeführt. Signifikante Ergebnisse $(p \leq 0,05)$ werden hervorgehoben.

\subsection{Fehler auf Wortebene}

Die Texte der Lernenden enthalten im Schnitt 10 richtige und knapp 7 falsch geschriebene Wörter, was einem Prozentsatz von etwa $60 \%$ richtig geschriebenen Wörtern entspricht. Mit durchschnittlich 4,26 O-Fehlern werden Wörter häufiger zwar lautgetreu, aber nicht orthografisch richtig geschrieben (z. B. ,ich kome “ statt „, ich komme“), wohingegen nur durchschnittlich 2,41 N-Fehler vorkommen, also Wörter, die nicht lautgetreu verschriftet sind (z. B. ,ich kone“ statt „,ich komme“). Die Unterschiede zwischen den einzelnen Lernenden fallen deutlich aus. So gibt es sowohl Lernende ohne O- oder N-Fehler als auch Kinder mit bis zu 24 O-Fehlern oder $16 \mathrm{~N}$-Fehlern. Sowohl für die Anzahl der richtig geschriebenen Wörter als auch für die Anzahl der „O-Fehler“ sind die Intraclass-Korrelationen relativ hoch. Der ICC = 0,20 für die Anzahl der richtig geschriebenen Wörter (vgl. Tab. 4) bedeutet beispielsweise, dass $20 \%$ der Unterschiede in der Anzahl richtig geschriebener Wörter auf Unterschiede zwischen den Klassen zurückzuführen sind.

Inwiefern sich „Lesen durch Schreiben“ auf die Fehler auf Wortebene auswirkt, ist in Tab. 5 abgebildet. Unter Kontrolle der Textlänge und der Vorläuferfertigkeiten „Anlaute hören“ und „Buchstabenkenntnis“ ergibt sich für die Anzahl der richtig geschriebenen Wörter (ohne Groß-/Kleinschreibung; 1.1) ein signifikant negativer Effekt von $\beta=-0,44^{*}(p \leq 0,05)$. Das heißt, je bedeutsamer die Lehrpersonen die Rolle von Reichen für ihren Unterricht einschätzen, desto weniger Wörter wer-

Tab. 4 Deskriptive Statistiken - Fehler auf Wortebene ( $N=507$ Lernende)

\begin{tabular}{lllllll}
\hline Item & & Min & Max & M & SD & ICC \\
\hline 1.1 & $\begin{array}{l}\text { Anzahl der richtig geschriebenen Wörter } \\
\text { (ohne Groß-/Kleinschreibung) }\end{array}$ & 1 & 73 & 10,00 & 7,23 & 0,20 \\
1.2 & $\begin{array}{l}\text { Anzahl der „O-Fehler“ (lautgetreue, aber } \\
\text { nicht orthografisch korrekte Wörter) } \\
1.3\end{array}$ & 0 & 24 & 4,26 & 3,67 & 0,22 \\
& $\begin{array}{l}\text { Anzahl der „N-Fehler“ (nicht lautgetreu } \\
\text { verschriftete Wörter) }\end{array}$ & 0 & 16 & 2,41 & 2,43 & 0,06 \\
\hline
\end{tabular}

Tab. 5 Mehrebenenanalysen - Fehler auf Wortebene ( $N=494$ Lernende)

\begin{tabular}{|c|c|c|c|c|c|c|c|c|c|c|}
\hline $\mathrm{AV}$ & & 1.1 & & & 1.2 & & & 1.3 & & \\
\hline UV & & $\beta$ & $p$ & $\mathrm{R}^{2}$ & $\beta$ & $p$ & $\mathrm{R}^{2}$ & $\beta$ & $p$ & $\mathrm{R}^{2}$ \\
\hline \multirow[t]{3}{*}{$\overline{\text { E1 }}$} & Textlänge & $\mathbf{0 , 8 3}$ & $\leq 0,001$ & 0,79 & 0,72 & $\leq 0,001$ & 0,51 & 0,51 & $\leq 0,001$ & $\overline{0,24}$ \\
\hline & Anlaute hören & $-0,03$ & 0,141 & & 0,14 & $\leq 0,001$ & & $-0,07$ & 0,173 & \\
\hline & Buchstabenkenntnis & $\mathbf{0 , 1 8}$ & $\leq 0,001$ & & $-0,18$ & $\leq 0,001$ & & $-0,20$ & $\leq 0,001$ & \\
\hline E2 & $\begin{array}{l}\text { Rolle von Rei- } \\
\text { chen }\end{array}$ & $-0,44$ & $\leq 0,05$ & 0,19 & 0,68 & $\leq 0,001$ & 0,46 & $-0,23$ & 0,325 & 0,05 \\
\hline
\end{tabular}


den von den Schülerinnen und Schülern insgesamt richtig geschrieben. Wie die beiden folgenden Modelle zeigen, ist dieser Unterschied auf die orthografischen Fehler zurückzuführen, also auf die zwar lautgetreu geschriebenen, aber nicht orthografisch korrekten Wörter. Je bedeutsamer Reichen für den Unterricht ist, desto mehr O-Fehler machen die Lernenden (1.2; $\left.\beta=0,68^{* * *} ; p \leq 0,001\right)$. Die Anzahl der $\mathrm{N}$-Fehler (1.3) ist hingegen nicht signifikant von der Bedeutsamkeit der Konzeption Lesen durch Schreiben abhängig $(\beta=-0,23 ; p=0,325)$. In der Tendenz machen Kinder, deren Lehrpersonen in ihrem Unterricht der Konzeption LdS eine größere Rolle beimessen, weniger Fehler in der lauttreuen Verschriftung. Mit Kontrolle der Lernvoraussetzungen und unter Berücksichtigung der Mehrebenstruktur ist dieser Zusammenhang aber nicht signifikant.

\subsection{OLFA-Stufen}

In der OLFA 1-2 wird von Phasen der Lernentwicklung ausgegangen, weshalb alle fehlerhaften Verschriftungen einer von drei Stufen zugeordnet werden können (Tab. 6). Die deskriptiven Statistiken zeigen hier zunächst, dass in allen drei Entwicklungsphasen deutliche interindividuelle Unterschiede zwischen den Kindern auftreten. Während insgesamt durchschnittlich 8,96 Fehler nach dem OLFA 1-2System gemacht werden, lassen sich davon durchschnittlich 3,19 Fehler der Stufe I zuordnen (rudimentäre Verschriftungen, z. B. Zeichenumstellungen, fehlerhafte Lautgliederung), 1,67 Fehler der Stufe II (annähernd lauttreue Schreibungen, z. B. „Hont“ für Hund) und 3,61 Fehler der Stufe III (lauttreue Verschriftungen mit orthografischen Elementen).

Für alle vier abhängigen Variablen zeigen sich unter Kontrolle der Variablen auf Ebene 1 generell Zusammenhänge in positiver Richtung mit der Unterrichts-

Tab. 6 Deskriptive Statistiken - OLFA-Stufen ( $N=507$ Lernende)

\begin{tabular}{lllllll}
\hline Item & & Min & Max & M & SD & ICC \\
\hline 2.1 & Fehleranzahl OLFA 1-2 & 0 & 43 & 8,96 & 6,76 & 0,11 \\
2.2 & Fehleranzahl OLFA 1-2 (Stufe I) & 0 & 19 & 3,19 & 3,21 & 0,07 \\
2.3 & Fehleranzahl OLFA 1-2 (Stufe II) & 0 & 15 & 1,67 & 2,16 & 0,07 \\
2.4 & Fehleranzahl OLFA 1-2 (Stufe III) & 0 & 18 & 3,61 & 3,05 & 0,13 \\
\hline
\end{tabular}

Tab. 7 Mehrebenenanalysen - OLFA-Stufen ( $N=494$ Lernende)

\begin{tabular}{|c|c|c|c|c|c|c|c|c|c|c|c|c|}
\hline AV & & 2.1 & & & 2.2 & & & 2.3 & & 2.4 & & \\
\hline UV & & $\beta$ & $p$ & $\mathrm{R}^{2}$ & $\beta$ & $p$ & $\mathrm{R}^{2}$ & $\beta$ & $\mathrm{R}^{2}$ & $\beta$ & $p$ & $\mathrm{R}^{2}$ \\
\hline \multirow[t]{3}{*}{ E1 } & Textlänge & 0,67 & $\leq 0,001$ & 0,40 & 0,38 & $\leq 0,001$ & 0,15 & 0,36 & $\leq \mathbf{0 , 0 0 1} 0,14$ & 0,68 & $\leq 0,001$ & 0,45 \\
\hline & $\begin{array}{l}\text { Anlaute } \\
\text { hören }\end{array}$ & 0,03 & 0,559 & & 0,01 & 0,874 & & $-0,01$ & 0,913 & 0,06 & 0,173 & \\
\hline & $\begin{array}{l}\text { Buchstaben- } \\
\text { kenntnis }\end{array}$ & $-0,26$ & $\leq 0,001$ & & $-0,26$ & $\leq 0,001$ & & $-0,26$ & $\leq 0,001$ & $-0,09$ & 0,121 & \\
\hline E2 & $\begin{array}{l}\text { Rolle } \\
\text { von } \\
\text { Reichen }\end{array}$ & 0,37 & 0,091 & 0,14 & 0,32 & 0,213 & 0,10 & 0,11 & $\begin{array}{lll}0,719 & 0,01\end{array}$ & 0,40 & $\leq 0,05$ & 0,16 \\
\hline
\end{tabular}


Tab. 8 Deskriptive Statistiken - Ausgewählte orthografische Fehler ( $N=507$ Lernende)

\begin{tabular}{lllllll}
\hline Item & & Min & Max & M & SD & ICC \\
\hline 3.1 & $\begin{array}{l}\text { Einfachschreibung für Konsonantenver- } \\
\text { doppelung }\end{array}$ & 0 & 12 & 1,04 & 1,31 & 0,10 \\
3.2 & $\begin{array}{l}\text { Einfachschreibung für markierte Vokal- } \\
\text { länge }\end{array}$ & 0 & 4 & 0,33 & 0,61 & 0,10 \\
3.3 & i für ie bei langem /i:/ & 0 & 6 & 0,63 & 0,89 & 0,09 \\
3.4 & ie für i bei langem /i:/ & 0 & 4 & 0,11 & 0,37 & 0,09 \\
3.5 & $\begin{array}{l}\text { Fehlender Schwa-Laut am Ende eines } \\
\text { Verbs 1. Person Singular Präsens }\end{array}$ & 0 & 3 & 0,14 & 0,38 & 0,05 \\
\hline
\end{tabular}

konzeption, d.h. je größer die Rolle von Reichen, desto mehr Fehler machen die Schülerinnen und Schüler. Nur für Fehler der Stufe III (2.4) ist dieser Zusammenhang aber signifikant $\left(\beta=0,40^{*} ; p \leq 0,05\right)$, für die Gesamtanzahl der Fehler nach OLFA 1-2 (2.1) zeigt sich eine Tendenz $(\beta=0,37 ; p \leq 0,10)$ (Tab. 7).

\subsection{Ausgewählte orthografische Fehler}

Auch bei den ausgewählten orthografischen Fehlern gibt es sowohl Kinder, die diese Fehler nie machen, als auch Kinder, bei denen sie gehäuft vorkommen. Im Vergleich ist der häufigste Fehler die „Einfachschreibung für Konsonantenverdoppelung“, den jedes Kind im Mittel 1,04-mal macht (Tab. 8).

Während die Bedeutsamkeit von LdS keinen signifikanten Effekt auf die „Einfachschreibung für Konsonantenverdoppelung (3.1)“ hat $(\beta=0,24 ; p=0,334)$, sind die Effekte für die „Einfachschreibung für markierte Vokallänge (3.2)“ $(\beta=0,64 * * *$; $p \leq 0,001$ ), für den ,fehlenden Schwa-Laut am Ende eines Verbs 1. Person Singular Präsens in der Reduktionssilbe $(3.5)^{\text {“ }}\left(\beta=0,53^{*} ; p \leq 0,05\right)$ signifikant und für ,i für ie bei langem /i:/ (3.3)“ tendenziell signifikant $(\beta=0,39 ; p=0,095)$. Hier machen Schülerinnen und Schüler also umso mehr Fehler, je größer die Bedeutsamkeit der Reichen-Konzeption laut Lehrperson ist. Ein umgekehrter Effekt ergibt sich für den Fehler ,,ie für i bei langem /i:/ (3.4)“ ( $\beta=-0,39 * ; p \leq 0,05)$ (Tab. 9).

\subsection{Groß- und Kleinschreibung}

Fehler in der Groß- und Kleinschreibung kommen am häufigsten durch „Großschreibungen im Wort“" zustande - der besonders hohe Maximalwert resultiert allerdings auch daraus, dass einige wenige Kinder komplett in Großbuchstaben geschrieben haben, was innerhalb von Klassen ähnlicher war als zwischen Klassen. Hier sind daher auch die ICCs sehr hoch (Tab. 10).

Während die „Klein- für Großschreibung (4.1)“ in keinem Zusammenhang zur Konzeption LdS steht $(\beta=-0,05 ; p=0,833)$, machen Kinder, in deren Unterricht LdS eine größere Rolle spielt, signifikant mehr Fehler der Kategorien „Groß- für Kleinschreibung (4.2)“ ( $\beta=0,44 * * * ; p \leq 0,001)$ und ,Großschreibung im Wort (4.3)“ $(\beta=0,47 * * * ; p \leq 0,001)$ (Tab. 11). 


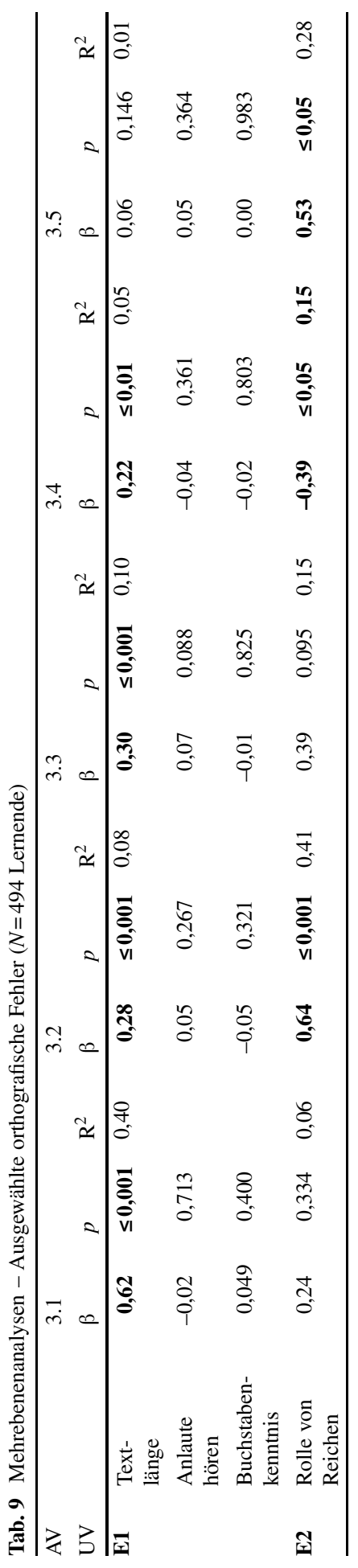


Tab. 10 Deskriptive Statistiken - Groß- und Kleinschreibung ( $N=507$ Lernende)

\begin{tabular}{lllllll}
\hline Item & & Min & Max & M & SD & ICC \\
\hline 4.1 & Klein- für Großschreibung & 0 & 5 & 0,51 & 0,86 & 0,09 \\
4.2 & Groß- für Kleinschreibung & 0 & 54 & 2,94 & 4,93 & 0,40 \\
4.3 & Großschreibung im Wort & 0 & 242 & 6,82 & 22,49 & 0,63 \\
\hline
\end{tabular}

Tab. 11 Mehrebenenanalysen - Groß- und Kleinschreibung ( $N=494$ Lernende)

\begin{tabular}{|c|c|c|c|c|c|c|c|c|c|c|}
\hline AV & & 4.1 & & & 4.2 & & & 4.3 & & \\
\hline UV & & $\beta$ & $p$ & $\mathrm{R}^{2}$ & $\beta$ & $p$ & $\mathrm{R}^{2}$ & $\beta$ & $p$ & $\mathrm{R}^{2}$ \\
\hline \multirow[t]{3}{*}{$\overline{\mathbf{E 1}}$} & Textlänge & 0,37 & $\leq 0,001$ & 0,14 & 0,49 & $\leq 0,001$ & 0,24 & 0,36 & $\leq 0,05$ & 0,12 \\
\hline & $\begin{array}{l}\text { Anlaute } \\
\text { hören }\end{array}$ & 0,01 & 0,863 & & $-0,01$ & 0,909 & & $-0,05$ & 0,105 & \\
\hline & $\begin{array}{l}\text { Buchstaben- } \\
\text { kenntnis }\end{array}$ & 0,02 & 0,700 & & 0,00 & 0,942 & & $-0,02$ & 0,730 & \\
\hline E2 & $\begin{array}{l}\text { Rolle von } \\
\text { Reichen }\end{array}$ & $-0,05$ & 0,833 & 0,00 & 0,44 & $\leq 0,001$ & 0,20 & 0,47 & $\leq 0,001$ & 0,22 \\
\hline
\end{tabular}

\subsection{Getrennt- und Zusammenschreibung}

Die häufigste Fehlerart im Bereich der Getrennt- und Zusammenschreibung ist die „Zusammen- für Getrenntschreibung“. Die Klassenzugehörigkeit spielt insbesondere für die Fehler „Getrennt- für Zusammenschreibung“ $(\mathrm{ICC}=0,17)$ und „Platzhalter zwischen Wörtern fehlt" (ICC=0,21) eine bedeutsame Rolle (Tab. 12).

Während die Konzeption LdS keine Bedeutung für die Fehlerhäufigkeit im Bereich „Getrennt- für Zusammenschreibung (5.1)“ hat ( $\beta=-0,06 ; p=0,775)$, machen in den übrigen drei Fehlerbereichen die Kinder signifikant mehr Fehler, wenn die Rolle von Reichen für den Unterricht als bedeutsamer eingeschätzt wird (Tab. 13).

\subsection{Interpunktion}

Im Mittel machen die Erstklässlerinnen und Erstklässler 1,15 Fehler bei der Verwendung der Satzabschlusszeichen und 0,57 Fehler bei den Satzmittezeichen (hier v. a. Kommata). Dabei ist zu berücksichtigen, dass die Texte durchschnittlich aus knapp zwei Sätzen bestehen. Die Intraclass-Korrelationen deuten auf die Wichtigkeit der Klassenzugehörigkeit bei der Interpunktion hin (Tab. 14).

Tab. 12 Deskriptive Statistiken - Getrennt- und Zusammenschreibung ( $N=507$ Lernende)

\begin{tabular}{lllllll}
\hline Item & & Min & Max & M & SD & ICC \\
\hline 5.1 & Getrennt- für Zusammenschreibung & 0 & 3 & 0,30 & 0,64 & 0,17 \\
5.2 & Zusammen- für Getrenntschreibung & 0 & 15 & 0,76 & 1,55 & 0,07 \\
5.3 & Getrenntschreibung von unselbstständi- & 0 & 8 & 0,22 & 0,63 & 0,07 \\
& gen Teilen & & & & & \\
5.4 & Platzhalter zwischen Wörtern fehlt & 0 & 4 & 0,27 & 0,91 & 0,21 \\
\hline
\end{tabular}


Tab. 13 Mehrebenenanalysen - Getrennt- und Zusammenschreibung ( $N=494$ Lernende)

\begin{tabular}{|c|c|c|c|c|c|c|c|c|c|c|c|c|c|}
\hline AV & & 5.1 & & & 5.2 & & & 5.3 & & & 5.4 & & \\
\hline UV & & $\beta$ & $p$ & $\mathrm{R}^{2}$ & $\beta$ & $p$ & $\mathrm{R}^{2}$ & $\beta$ & $p$ & $\mathrm{R}^{2}$ & $\beta$ & $p$ & $\mathrm{R}^{2}$ \\
\hline \multirow[t]{3}{*}{ E1 } & Textlänge & 0,22 & $\leq 0,01$ & 0,07 & 0,30 & $\leq 0,001$ & 0,09 & 0,17 & $\leq 0,01$ & 0,03 & 0,07 & 0,423 & 0,01 \\
\hline & $\begin{array}{l}\text { Anlaute } \\
\text { hören }\end{array}$ & 0,03 & 0,428 & & 0,02 & 0,617 & & 0,03 & 0,532 & & $-0,02$ & 0,582 & \\
\hline & $\begin{array}{l}\text { Buchstaben- } \\
\text { kenntnis }\end{array}$ & 0,05 & 0,554 & & $-0,20$ & $\leq 0,001$ & & $-0,12$ & $\leq 0,05$ & & $-0,10$ & $\leq 0,01$ & \\
\hline E2 & $\begin{array}{l}\text { Rolle von } \\
\text { Reichen }\end{array}$ & $-0,06$ & 0,775 & 0,00 & 0,91 & $\leq 0,001$ & 0,83 & 0,79 & $\leq 0,001$ & 0,62 & $\mathbf{0 , 5 8}$ & $\leq 0,001$ & 0,34 \\
\hline
\end{tabular}

Tab. 14 Deskriptive Statistiken - Interpunktion ( $N=507$ Lernende)

\begin{tabular}{lllllll}
\hline Item & & Min & Max & M & SD & ICC \\
\hline 6.1 & $\begin{array}{l}\text { Fehleranzahl Satzabschlusszeichen } \\
\text { gesamt }\end{array}$ & 0 & 11 & 1,15 & 1,29 & 0,22 \\
6.2 & $\begin{array}{l}\text { Fehleranzahl Satzmittezeichen ge- } \\
\text { samt }\end{array}$ & 0 & 5 & 0,57 & 0,84 & 0,12 \\
6.3 & Fehleranzahl Interpunktion gesamt & 0 & 12 & 1,72 & 1,68 & 0,23 \\
\hline
\end{tabular}

Tab. 15 Mehrebenenanalysen - Interpunktion ( $N=494$ Lernende)

\begin{tabular}{|c|c|c|c|c|c|c|c|c|c|c|}
\hline \multirow{2}{*}{$\begin{array}{l}\text { AV } \\
\mathrm{UV}\end{array}$} & & \multicolumn{3}{|l|}{6.1} & \multicolumn{3}{|l|}{6.2} & \multicolumn{3}{|l|}{6.3} \\
\hline & & $\beta$ & $p$ & $\mathrm{R}^{2}$ & $\beta$ & $p$ & $\mathrm{R}^{2}$ & $\beta$ & $p$ & $\mathrm{R}^{2}$ \\
\hline \multirow[t]{3}{*}{$\overline{\text { E1 }}$} & Textlänge & 0,55 & $\leq 0,001$ & 0,27 & 0,63 & $\leq 0,001$ & 0,40 & 0,74 & $\leq 0,001$ & 0,51 \\
\hline & $\begin{array}{l}\text { Anlaute } \\
\text { hören }\end{array}$ & $-0,01$ & 0,813 & & 0,03 & 0,399 & & 0,01 & 0,767 & \\
\hline & $\begin{array}{l}\text { Buchstaben- } \\
\text { kenntnis }\end{array}$ & $-0,10$ & 0,064 & & $-0,01$ & 0,787 & & $-0,08$ & $\leq 0,05$ & \\
\hline E2 & $\begin{array}{l}\text { Rolle von } \\
\text { Reichen }\end{array}$ & 0,38 & 0,051 & 0,14 & $-0,01$ & 0,953 & 0,00 & 0,36 & 0,080 & 0,13 \\
\hline
\end{tabular}

Bei den Fehlern in der Verwendung der Satzmittezeichen (6.2) zeigt sich keinerlei Effekt der Konzeption LdS. Bei den Fehlern in den Satzabschlusszeichen (6.1) und bei der Interpunktion insgesamt (6.3) deuten die positiven Koeffizienten in der Tendenz $(p \leq 0,10)$ Nachteile für Kinder an, in deren Unterricht die Rolle von LdS stärker ausgeprägt ist (Tab. 15).

\section{Zusammenfassung, Diskussion und Ausblick}

Insgesamt können die aufgestellten Hypothesen weitgehend bestätigt werden. In einem Unterricht, in dem die Konzeption „Lesen durch Schreiben“ laut Lehrpersonen eine stärkere Rolle spielt, machen die Lernenden in der Mitte des ersten Schuljahres unter Kontrolle von Textlänge und Vorläuferfertigkeiten auf Wort- und Graphemebene sowie bei der Groß- und Kleinschreibung, der Getrennt- und Zusammenschreibung und der Interpunktion insgesamt mehr Fehler als in einem Unterricht, in dem der Konzeption eine geringere Bedeutung zukommt. Dabei lassen sich die höheren 
Fehleranzahlen auf orthografisch fehlerhafte Verschriftungen zurückführen, nicht aber auf Fehler in der lauttreuen Verschriftung. Vereinzelt gibt es auch Subkategorien, in denen keine Unterschiede in Abhängigkeit von der Unterrichtskonzeption festzustellen sind (z.B. „Einfachschreibung für Konsonantenverdoppelung“, „Getrenntfür Zusammenschreibung“). Da nach Reichen im ersten Schuljahr orthografische Regeln nicht vermittelt und orthografische Fehler normalerweise nicht korrigiert werden, überrascht es wenig, dass Kinder, in deren Unterricht die Reichen-Konzeption ein stärkeres Gewicht einnimmt, mehr orthografische Fehler machen. Vorteile im lautorientierten Schreiben können allerdings auch nicht festgestellt werden.

Ein einziger signifikanter ,,Vorteil“ von Kindern, deren Lehrpersonen der Konzeption von Reichen eine höhere Bedeutung für ihren Unterricht beimessen, ergibt sich für die Kategorie ,,ie für i bei langem /i:/“. Das bedeutet, dass Schülerinnen und Schüler, deren Unterricht sich stärker an der Reichen-Konzeption orientiert, seltener die Schreibweise <ie> wählen, wenn ein Wort ein /i:/ enthält, also mit einem langen Vokal gesprochen wird, aber orthografisch korrekt dennoch mit einfachem $<$ i $>$ geschrieben wird - sie schreiben also beispielsweise seltener „Krokodiel“" oder „mier“. Dieser augenscheinliche Vorteil kommt wahrscheinlich dadurch zustande, dass Schülerinnen und Schüler, in deren Unterricht LdS eine größere Bedeutung zukommt, zunächst alle Wörter mit $<\mathrm{i}>$ schreiben, da sie das $<$ ie $>$ oder $<$ ih $>$ vermutlich noch nicht kennengelernt haben. Kinder in einem Unterricht, in dem LdS eine geringere Rolle spielt, haben hingegen wahrscheinlich gelernt, dass das /i:/ in den meisten Fällen als <ie> verschriftet wird, sodass sie diese Regel dann auch bei Wörtern anwenden, auf die sie (ausnahmsweise) nicht zutrifft. Für das /i:/ ist die Verschriftung als <ie> nämlich das Basisgraphem (Thomé und Thomé 2019), d.h. diese Wörter werden in den meisten Fällen (72\%) mit dem Graphem <ie> verschriftet $(18 \%<\mathrm{ih}>, 9 \%<\mathrm{i}>; 1 \%<\mathrm{ieh}>)$. Empfohlen wird daher auch, dass die Kinder zunächst die häufigste Verschriftung kennenlernen sollten, also das Basisgraphem, und Ausnahmen erst später hinzukommen sollten (Thomé und Thomé 2014). Insgesamt verdeutlichen die qualitativen Fehleranalysen, dass für einen guten schriftsprachlichen Unterricht das linguistische Wissen der Lehrkraft bedeutsam ist, um augenscheinliche „Fehler“ von Schülerinnen und Schülern richtig einordnen zu können und sich bewusst für einen didaktisch sinnvollen Umgang mit ihnen zu entscheiden.

Obwohl sich der Rechtschreiberwerb über mehrere Jahre erstreckt und es für die Beurteilung der Wirksamkeit der Reichen-Konzeption daher natürlich wichtig ist, auch den Verlauf in den weiteren Schuljahren zu betrachten, stellt sich die Frage, ob bereits im ersten Schuljahr Anregungen in Richtung Orthografie sinnvoll sind - was die Ergebnisse der vorliegenden Studie nahelegen. Dass sich in der vorliegenden Studie die Unterschiede je nach verwendeter Konzeption nämlich schon so früh (nach etwa sechs Monaten Schulbesuch) und teilweise deutlich nachweisen lassen, zeigt, dass es Lehrpersonen durchaus früh gelingen kann, erste schriftsprachliche Normen in die Wahrnehmung zu rücken, die von den Kindern in freien Texten angewendet werden können. Wenn sich in einzelnen orthografischen Bereichen keine/sehr geringe Unterschiede zeigen, könnte das daran liegen, dass hier unabhängig von der verwendeten schriftsprachlichen Konzeption noch keine Regelvermittlung stattfand (wie hier beispielsweise bei den Satzmittezeichen, die von den Kindern insgesamt 
noch kaum (richtig) verwendet werden, sodass auch keine Unterschiede je nach Konzeption feststellbar sind). Corvacho del Toro und Hoffmann-Erz (2014) stellen in ihrer Auseinandersetzung mit methodischen Unterrichtskonzepten des Schriftspracherwerbs als Fazit heraus, dass weder das Verharren über einen langen Zeitraum beim rein lautorientierten Schreiben zu empfehlen ist noch das Verbessern aller Fehler von Anfang an, da hier die Schülerinnen und Schüler ,zu viele Informationen unstrukturiert" erhalten würden (Corvacho del Toro und Hoffmann-Erz 2014, S. 37).

Zuletzt sollen besondere Chancen, aber auch Grenzen der vorliegenden Studie kurz diskutiert werden. Zunächst ist die Stichprobe mit 31 Klassen und 507 Schülerinnen und Schülern ausreichend groß, um zu aussagekräftigen Ergebnissen zu gelangen, wenngleich die Zusammensetzung der Stichprobe nicht repräsentativ ist, da der mittlere sozioökonomische Status der an der Studie teilnehmenden Schülerinnen und Schüler höher liegt als der gesamtdeutsche Durchschnitt (vgl. Lipowsky et al. 2018). Des Weiteren muss bei der Interpretation der Ergebnisse berücksichtigt werden, dass die Schülertexte nicht in einer standardisierten Testsituation erhoben wurden. Interessante Aufschlüsse bietet aber die Untersuchung freier Texte anhand des OLFA-Systems, bei dem unterschiedliche Fehlerarten differenziert auf Graphemebene ausgewertet werden. Allerdings sind die hier untersuchten Texte mit durchschnittlich 17 Wörtern (SD=9,82) wesentlich kürzer als 100 Wörter. Dies schränkt die Aussagekraft über einen möglichen Förderbedarf ein, was aber ohnehin nicht das Ziel der vorliegenden Untersuchung ist. Eine entscheidende Verbesserung gegenüber einigen Studien ist aber die Kontrolle der schriftsprachlichen Vorläuferfertigkeiten und die Berücksichtigung der Mehrebenstruktur der Daten.

Die Ergebnisse ermöglichen auch zahlreiche Anknüpfungspunkte für weitere Auswertungen. Ein erster Schritt besteht dabei in der genaueren Analyse von Unterrichtstagebüchern und Unterrichtsvideos hinsichtlich der tatsächlichen Umsetzung der verschiedenen Aspekte des Reichen-Ansatzes, was dazu dienen soll, die Angaben der Lehrpersonen zu ihrem Unterricht noch breiter zu validieren. Dies ist bedeutsam, da beispielsweise Peschel (2004) in seiner Beobachtungsstudie zweier Klassen aufzeigte, dass die Selbsteinschätzung der Lehrkräfte hinsichtlich der Umsetzung des Reichen-Ansatzes teilweise zu Fehleinschätzungen führen kann und mit der Außensicht nicht übereinstimmen muss. Erste weiterführende Analysen deuten aber bereits darauf hin, dass die Angaben der Lehrpersonen zur Rolle der ReichenKonzeption erwartungsgemäß mit weiteren Aspekten ihres Unterrichts zusammenhängen.

In weiterführenden Beiträgen soll außerdem die Entwicklung der standardisiert erfassten Rechtschreibleistung und des Leseverständnisses, aber auch des Selbstkonzepts in Abhängigkeit von der Bedeutung der Konzeption „Lesen durch Schreiben“ über das erste Schuljahr hinaus längsschnittlich analysiert werden, um auch die Frage nach den langfristigen Wirkungen beantworten zu können. Außerdem sollen differenzielle Effekte der Konzeption für leistungsstarke und -schwache Schülerinnen und Schüler fokussiert und die Rolle der Klassenzusammensetzung und der Unterrichtsqualität näher beleuchtet werden. Auch die Untersuchung von Effekten der Unterrichtskonzeption auf die Länge der Texte nach Kontrolle der Schreibzeit sowie auf die inhaltliche Textqualität stehen im Fokus von weiterführenden Analysen. 
Interessant könnten auch Analysen zum Schreibwortschatz der Kinder sein und zur Frage, ob sich dieser in Abhängigkeit von der Unterrichtskonzeption unterscheidet.

Funding Open Access funding provided by Projekt DEAL.

Open Access Dieser Artikel wird unter der Creative Commons Namensnennung 4.0 International Lizenz veröffentlicht, welche die Nutzung, Vervielfältigung, Bearbeitung, Verbreitung und Wiedergabe in jeglichem Medium und Format erlaubt, sofern Sie den/die ursprünglichen Autor(en) und die Quelle ordnungsgemäß nennen, einen Link zur Creative Commons Lizenz beifügen und angeben, ob Änderungen vorgenommen wurden.

Die in diesem Artikel enthaltenen Bilder und sonstiges Drittmaterial unterliegen ebenfalls der genannten Creative Commons Lizenz, sofern sich aus der Abbildungslegende nichts anderes ergibt. Sofern das betreffende Material nicht unter der genannten Creative Commons Lizenz steht und die betreffende Handlung nicht nach gesetzlichen Vorschriften erlaubt ist, ist für die oben aufgeführten Weiterverwendungen des Materials die Einwilligung des jeweiligen Rechteinhabers einzuholen.

Weitere Details zur Lizenz entnehmen Sie bitte der Lizenzinformation auf http://creativecommons.org/ licenses/by/4.0/deed.de.

\section{Literatur}

Augst, G., \& Dehn, M. (2013). Rechtschreibung und Rechtschreibunterricht. Stuttgart: Klett.

Bredel, U., Fuhrhop, N., \& Noack, C. (2011). Wie Kinder lesen und schreiben lernen. Tübingen: Narr.

Brügelmann, H., Hengartner, E., \& Reichen, J. (1994a). Richtig schreiben durch freies Schreiben? Rechtschreibentwicklung in Schweizer Klassen, die nach „Lesen durch Schreiben“ unterrichtet wurden. In H. Brügelmann \& S. Richter (Hrsg.), Wie wir recht schreiben lernen. 10 Jahre Kinder auf dem Weg zur Schrift (S. 135-148). Lengwil am Bodensee: Libelle.

Brügelmann, H., Lange, I., \& Spitta, G. (1994b). Schreibvergleich BRDDR 1990/91. In H. Brügelmann \& S. Richter (Hrsg.), Wie wir recht schreiben lernen. 10 Jahre Kinder auf dem Weg zur Schrift (S. 129-134). Lengwil am Bodensee: Libelle.

Brügelmann, H. (2020). Einordnung der Ergebnisse der „Bonner Studie“ zur Wirkung verschiedener Ansätze des Lese- und Schreibunterrichts auf die Entwicklung der Rechtschreibleistung im Verlauf der Grundschulzeit. https://t1p.de/bonner-studie-komm. Zugegriffen: 23. Mai 2020.

Corvacho del Toro, I., \& Hoffmann-Erz, R. (2014). Was ist lautgetreu? Zur Notwendigkeit einer begrifflichen Differenzierung. In K. Siekmann (Hrsg.), Theorie, Empirie und Praxis effektiver Rechtschreibdiagnostik (S. 29-40). Tübingen: Stauffenberg.

Ditton, H. (1998). Mehrebenenanalyse. Grundlagen und Anwendungen des hierarchisch linearen Modells. Weinheim: Juventa.

Doucet, S. A., \& Wilsdorf, A. (2005). Lucy rettet Mama Kroko. Hamburg: Oetinger.

Dürscheid, C. (2011). „Schreib nicht, wie du sprichst.“ Ein Thema für den Deutschunterricht. In B. Rothstein (Hrsg.), Sprachvergleich in der Schule. Thema Sprache - Wissenschaft für den Unterricht (S. 89-109). Baltmannsweiler: Schneider.

Friedrich, K. (2010). Unterrichtskonzept und Schriftspracherwerb. Dissertation. Heidelberg: Pädagogische Hochschule Heidelberg. https://opus.ph-heidelberg.de/frontdoor/deliver/index/docId/28/file/ Friedrich_Dissertation_03_08_2010.pdf. Zugegriffen: 24.07.2020.

Frith, U. (1986). Psychologische Aspekte des orthografischen Wissens. In G. Augst (Hrsg.), New trends in Graphemics and orthography (S. 218-233). Berlin: de Gruyter.

Funke, R. (2014). Erstunterricht nach der Methode ,Lesen durch Schreiben“ und Ergebnisse schriftsprachlichen Lernens. Eine metaanalytische Bestandsaufnahme. Didaktik Deutsch, 19, 20-41.

Gresser, A., Pohl, K., Corvacho del Toro, I., Greb, K., \& Faust, G. (2009). Modul 2 - Deutsch. In F. Lipowsky, G. Faust \& K. Greb (Hrsg.), Dokumentation der Erhebungsinstrumente des Projekts „Persönlichkeits- und Lernentwicklung von Grundschulkindern“ (PERLE). 1. PERLE-Instrumente: Schüler, Lehrer, Eltern (S. 34-40). Frankfurt a. M.: GFPF.

Hanke, P. (2005). Öffnung des Unterrichts in der Grundschule. Lehr-Lernkulturen und orthografische Lernprozesse im Vergleich. Münster: Waxmann. 
Hess, M., Denn, A.-K., \& Kirschhock, E. (in Vorbereitung). Wie wird der Rechtschreibunterricht in der ersten Jahrgangsstufe gestaltet? In M. Hess, A.-K. Denn, C. Theurer \& F. Lipowsky (Hrsg.), Determinanten und Effekte der Persönlichkeits- und Lernentwicklung in der Grundschule. Wiesbaden: VS.

Heuß, G. (1993). Erstlesen und Erstschreiben. Donauwörth: Auer.

Hüttis-Graff, P. (1997). Schriftorientierung im Unterricht. Rechtschreibenlernen unter den Bedingungen von Mehrsprachigkeit. Grundschulzeitschrift, 11, 48-53.

Jaeuthe, J., Bogda, K., Bosse, S., Lambrecht, J., \& Spörer, N. (2019). Entwickelt sich die Rechtschreibkompetenz von Grundschulkindern theoriekonform? In DGfE-Tagung „Eine Schule für alle - 100 Jahre Grundschule. Mythen, Widersprüche, Gewissheiten“. Erfurt, 26. Sept. 2019. Poster.

Kirschhock, E.-M. (2004). Entwicklung schriftsprachlicher Kompetenzen im Anfangsunterricht. Bad Heilbrunn: Klinkhardt.

Krauß, A. (2014). Schriftspracherwerb als Orthographieerwerb. Reflexionen - Realisationen - Relationen - Rekapitulationen. Baltmannsweiler: Schneider.

Kuhl, T. (2020). Rechtschreibung in der Grundschule. Eine empirische Untersuchung der Auswirkungen verschiedener Unterrichtsmethoden. Wiesbaden: VS.

Kuhl, T., \& Röhr-Sendlmeier, U. (2018). Rechtschreiberfolg nach unterschiedlichen Didaktiken. Weitblick, 2, 6-9.

Kürzinger, A., Lotz, M., Gleich, A.-K., \& Kempter, I. (2013). Auswertung der Lucybriefe: Perspektivenübernahme und Schreibkompetenz. In M. Lotz, F. Lipowsky \& G. Faust (Hrsg.), Technischer Bericht zu den PERLE-Videostudien (S. 255-296). Frankfurt a. M.: GFPF.

Landerl, K., Wimmer, H., \& Moser, E. (2006). SLRT. Salzburger Lese- und Rechtschreibtest. Verfahren zur Differentialdiagnose von Störungen des Lesens und Schreibens für die 1. bis 4. Schulstufe. Bern: Huber.

Lipowsky, F., Faust, G., \& Kastens, C. (2013). Persönlichkeits- und Lernentwicklung an staatlichen und privaten Grundschulen: Ergebnisse der PERLE-Studie zu den ersten beiden Schuljahren. Münster: Waxmann.

Lipowsky, F., Stubbe, T. C., Theurer, C., \& Faust, G. (2018). Wer liegt am Ende der Grundschulzeit vorn? Leistungsentwicklung von Schülerinnen und Schülern an privaten BIP-Kreativitätsgrundschulen und an staatlichen Grundschulen. Zeitschrift für Erziehungswissenschaft, 21, 897-927.

Lorenz, A. (2017). Wer schreibt recht, wer schreibt schlecht? Eine Untersuchung des Zusammenhangs zwischen der Rechtschreibleistung von Erstklässlern und dem Ansatz, „Lesen durch Schreiben“ von Jürgen Reichen in der Videostudie Deutsch des Projekts PERLE. Kassel: University press.

Lotz, M., \& Corvacho del Toro, I. (2013). Die Videostudie im Fach Deutsch: „Lucy rettet Mama Kroko“. In M. Lotz, F. Lipowsky \& G. Faust (Hrsg.), Technischer Bericht zu den PERLE-Videostudien (S. 29-36). Frankfurt a. M.: GFPF.

Lotz, M., Schoreit, E., \& Kemper, I. (2013). Die Entwicklung des Leseverständnisses und der Rechtschreibfähigkeit. In F. Lipowsky, G. Faust \& C. Kastens (Hrsg.), Persönlichkeits- und Lernentwicklung an staatlichen und privaten Grundschulen: Ergebnisse der PERLE-Studie zu den ersten beiden Schuljahren (S. 77-98). Münster: Waxmann.

May, P. (2001). Lernförderlicher Unterricht. Teil 1: Untersuchung zur Wirksamkeit von Unterricht und Förderunterricht für den schriftsprachlichen Lernerfolg. Frankfurt a. M.: Peter Lang.

Muthén, L.K., \& Muthén, B.O. (2012). Mplus. Los Angeles, California: Muthén \& Muthén. Computer software

Peschel, M. (2004). Lesen durch Schreiben in offenen Lernsituationen. Eine qualitative Fallstudie in Hamburger ersten Klassen zum Konzept „Lesen durch Schreiben“ von Jürgen Reichen. Berlin: wbv.

Reichen, J. (1988a). Allgemeindidaktische und organisatorische Empfehlungen. Lesen durch Schreiben. Zürich: Sabe.

Reichen, J. (1988b). Wie Kinder selbstgesteuert lesen lernen. Lesedidaktische, lernpsychologische und schulpädagogische Grundlagen eines vom Schüler selbstgesteuerten Schriftspracherwerbs. Lesen durch Schreiben. Zürich: Sabe.

Reichen, J. (2003). Hannah hat Kino im Kopf. Hamburg: Heinevetter.

Röber-Siekmeyer, C., \& Spiekermann, H. (2000). Die Ignorierung der Linguistik in der Theorie und Praxis des Schriftspracherwerbs. Überlegungen zu einer Neubestimmung des Verhältnisses von Pädagogik und Phonetik/Phonologie. Zeitschrift für Pädagogik, 46, 753-771.

Scheerer-Neumann, G. (1986). Wortspezifisch JA- Wortbild NEIN. Ein letztes Lebewohl an die Wortbildtheorie. Teil 2: Rechtschreiben. In B. Brügelmann (Hrsg.), ABC und Schriftsprache: Rätsel für Kinder, Lehrer und Forscher (S. 171-185). Konstanz: Libelle. 
Scheerer-Neumann, G. (2010). Stufenmodell des Rechtschreiblernens. In Landesinstitut für Schule und Medien Berlin-Brandenburg (Hrsg.), Individuelle Lernstandsanalysen Rechtschreiben. Lehrerheft. ILeA, (Bd. 2, S. 12-14). Ludwigsfelde: Landesinstitut für Schule und Medien Berlin-Brandenburg.

Scheerer-Neumann, G. (2017). Anmerkungen zu Stufenmodellen des Schriftspracherwerbs. In K. Siekmann, I. Corvacho del Toro \& R. Hoffmann-Erz (Hrsg.), Schriftsprachliche Kompetenzen in Theorie und Praxis (S. 73-85). Tübingen: Stauffenberg.

Schründer-Lenzen, A. (2007). Schriftspracherwerb und Unterricht. Bausteine professionellen Handlungswissens. Wiesbaden: VS.

Schründer-Lenzen, A., \& Merkens, H. (2006). Differenzen schriftsprachlicher Kompetenzentwicklung bei Kindern mit und ohne Migrationshintergrund. In A. Schründer-Lenzen (Hrsg.), Risikofaktoren kindlicher Entwicklung (S. 15-44). Wiesbaden: VS.

Thomé, D., \& Thomé, G. (2014). Basisgrapheme. Oldenburg: Institut für sprachliche Bildung.

Thomé, G., \& Thomé, D. (2010). OLFA 3-9. Oldenburger Fehleranalyse für die Klassen 3-9. Oldenburg: Institut für sprachliche Bildung.

Thomé, G., \& Thomé, D. (2011). OLFA 1-2. Oldenburger Fehleranalyse für die Klassen 1 und 2. Oldenburg: Institut für sprachliche Bildung.

Thomé, G., \& Thomé, D. (2016). Deutsche Wörter nach Laut- und Schrifteinheiten gegliedert. Oldenburg: Institut für sprachliche Bildung.

Thomé, G., \& Thomé, D. (2019). OLFA 1-2. Oldenburg: Institut für sprachliche Bildung.

Weinhold, S. (2006). Entwicklungsverläufe im Lesen- und Schreibenlernen in Abhängigkeit verschiedener didaktischer Konzepte. Eine Longitudinalstudie in Klasse 1-4. In S. Weinhold (Hrsg.), Schriftspracherwerb empirisch. Konzepte - Diagnostik - Entwicklung. Diskussionsforum Deutsch (S. 120-151). Baltmannsweiler: Schneider. 